Document downloaded from:

http://hdl.handle.net/10251/108671

This paper must be cited as:

Bozhko, A.; Sánchez-Dehesa Moreno-Cid, J.; Cervera Moreno, FS.; Krokhin, A. (2017).

Redirection and Splitting of Sound Waves by a Periodic Chain of Thin Perforated Cylindrical Shells. Physical Review Applied. 7(6):064034-1-064034-13.

doi:10.1103/PhysRevApplied.7.064034

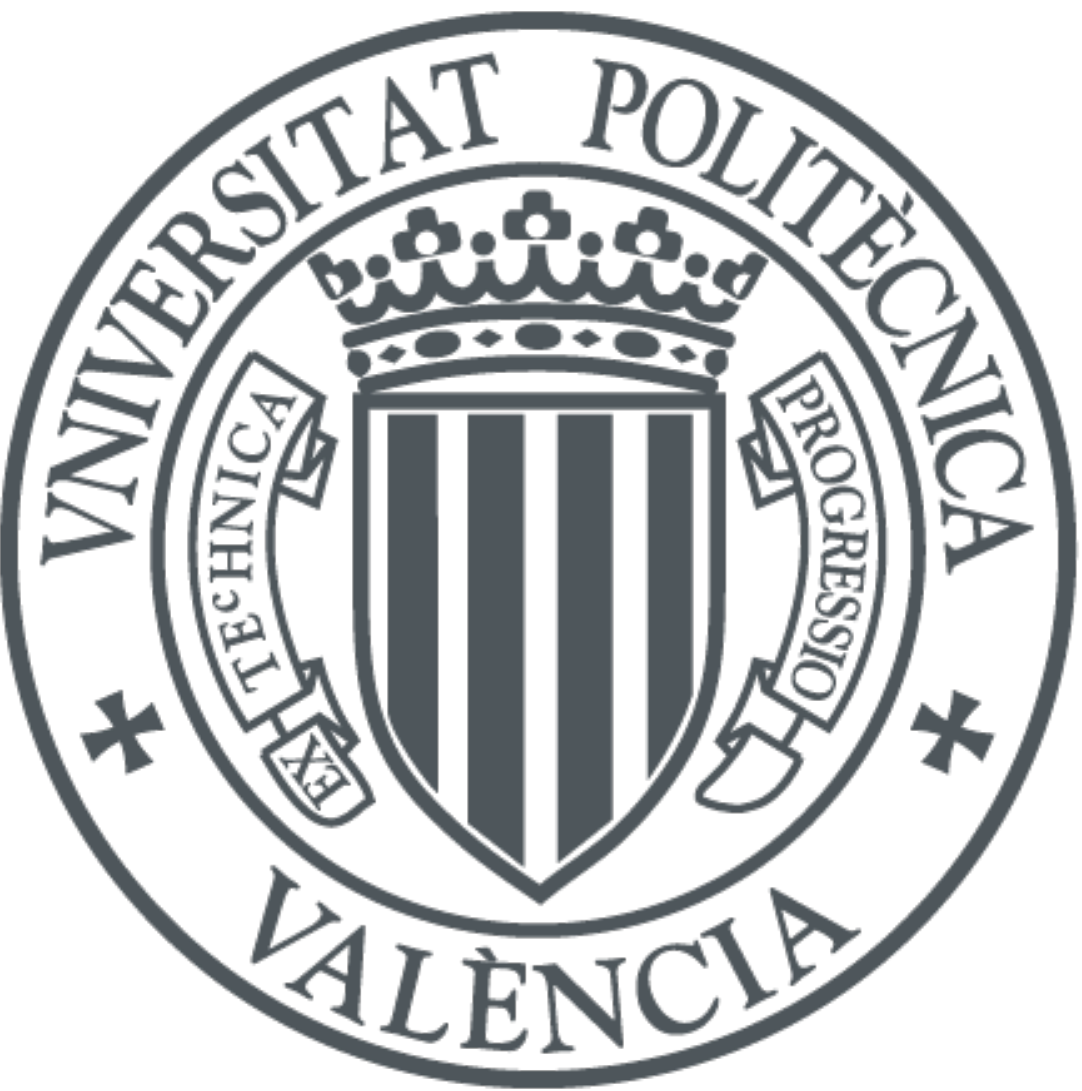

The final publication is available at

https://doi.org/10.1103/PhysRevApplied.7.064034

Copyright American Physical Society

Additional Information 


\title{
Redirection and splitting of sound waves by a periodic chain of thin perforated cylindrical shells
}

\author{
Andrey Bozhko, ${ }^{1}$ José Sánchez-Dehesa, ${ }^{2}$ Francisco Cervera, ${ }^{2}$ and Arkadii Krokhin ${ }^{1, *}$ \\ ${ }^{1}$ Department of Physics, University of North Texas, P. O. Box 311427, Denton, Texas 76203 \\ ${ }^{2}$ Wave Phenomena Group, Universitat Politècnica de València, \\ Camino de Vera s/n (Building $7 F$ ), E-46022 Valencia, Spain
}

(Dated: May 24, 2017)

\begin{abstract}
Scattering of sound by finite and infinite chains of equally spaced perforated metallic cylindrical shells in an ideal (inviscid) and viscous fluid is theoretically studied using rigorous analytical and numerical approaches. Due to perforations, a chain of thin shells is practically transparent for sound within a wide range of frequencies. It is shown that strong scattering and redirection of sound by $90^{\circ}$ may occur only for a discrete set of frequencies (Wood's anomalies) where the leaky eigenmodes are excited. The spectrum of eigenmodes consists of antisymmetric and symmetric branches with normal and anomalous dispersion, respectively. The antisymmetric eigenmode turns out to be a deaf mode since it cannot be excited at normal incidence. However, at slightly oblique incidence both modes can be excited at different but close frequencies. The symmetric mode, due to its anomalous dispersion, scatters sound in the "wrong" direction, thus allowing splitting of the incoming signal containing two harmonics into two beams propagating along the chain in the opposite directions. Calculations are presented for aluminum shells in viscous air where the effects of anomalous scattering, redirection, and signal splitting are well manifested.
\end{abstract}

PACS numbers: 43.20+g,43.30.+m,46.40.-f,42.25.Fx

Keywords: sound redirection, sound splitting, perforated shells, linear chain

\section{INTRODUCTION}

Periodic chains of highly symmetric scatterers, cylinders or spheres, may serve as waveguides for electromagnetic waves [1-3]. If the neighboring scatterers interact through the near-field plasmonic field, such a chain effectively transverses electromagnetic energy. In a linear chain, however, the Joule losses are accompanied by radiative [4-8] and collisionless nonradiative losses (Landau damping) [9]. In the case of acoustic waves, the interaction between two neighboring scatterers is much weaker due to the lack of an acoustic analog of gap plasmon resonance. However, in contrast with the dielectric permittivity, there are more possibilities to manipulate the density, elasticity, and viscosity of the scatterers, that allows one to predict and observe transport properties specific for sound waves.

Periodic chains of scatterers with internal structure (mass-in-mass units) may serve as a waveguide for mechanical energy, and also exhibit negative mass density [10]. Metamaterial behavior, where both the effective mass and the elastic modulus are negative, was predicted for a chain consisting of elastic mass-in-mass units with lateral resonances [11]. The dispersion curve of a chain of acoustic Helmholtz resonators (soda cans) with two scatterers per unit cell has a passing band within a hybridization gap where the effective index of refraction becomes negative [12]. These and other examples [13, 14] demonstrate that acoustic interactions between scatterers may

${ }^{*}$ Electronic address: Arkadii.Krokhin@unt.edu be sufficiently strong, leading to a variety of metamaterial properties.

At the same time, periodic structures with artificially weak scatterers are not less interesting since they allow observation of very delicate effects originating from interference between the scattering pattern of a single scatterer and collective wave motion resulting from periodicity [15]. Here, we would like to mention the so-called acoustic Poisson-like effect in the scattering of sound by periodic arrangement of cylindrical shells, predicted in Ref. [16]. This effect of $90^{\circ}$-redirection of acoustic energy becomes possible due to resonant excitation of an antisymmetric mode polarized in the direction perpendicular to the incoming sound. Periodic displacement of the cylindrical surface at the resonance resembles the Poisson effect in an elastic solid, which elongates under squeezing. Excitation of the antisymmetric mode occurs if the the strongest monopole and dipole terms in sound-matter interaction are switched off. This can be done by matching the density and elastic modulus of the solid shell to those of the background fluid $[17,18]$. As a result, the normally deaf antisymmetric collective mode is excited through the non-isotropic quadrupole term in the scattering cross-section, giving rise to propagation of sound waves in the direction perpendicular to incident wave [16]. Here, the weakness of scattering is not the physical reason of the Poisson-like effect itself, but it is the necessary condition for its observation.

A quite different physical reason leads to a similar effect of $90^{\circ}$-redirection of sound by a linear chain of perforated metallic cylindrical shells predicted in Ref. [19]. A perforated object serves as a weak scatterer if the radius of perforations $r$ greatly exceeds the thickness of the 
viscous layer $\delta=\sqrt{\eta_{0} / \rho_{0} \omega}$, where $\eta_{0}$ is the dynamic viscosity of the fluid, $\rho_{0}$ is the fluid density, and $\omega$ is the frequency of sound. Since a single perforated cylindrical shell is a weak scatterer without internal resonances, a periodic linear chain of perforated shells turns out to be almost transparent for sound at normal incidence [19]. However, anomalously low transmission was numerically predicted for the frequencies associated with the Wood's anomaly, which for this case occurs when the wavelength $\lambda$ becomes close to the period $d$ of the chain. Here, we propose the microscopic mechanism of this low transmission, which is due to resonant interaction of the external plane wave with a symmetric leaky eigenmode, and also explain why the condition $\lambda \approx d$ holds.

For this purpose, we develop a theory of scattering of external plane waves by a linear chain of perforated cylindrical shells with finite and infinite numbers of scatterers in viscous and ideal (inviscid) fluid. A method of expansion over cylindrical waves applied here leads to an infinite set of linear equations for partial transmission and scattering amplitudes. Also, a transcendental equation for the dispersion law of the eigenmodes is derived and numerically solved for the few lowest bands. Because of weak scattering, the band structure is close to that obtained in the empty-lattice model. Away from points of degeneracy, the dispersion is practically linear with speed equal to the speed of sound in the background fluid. However, at the $\Gamma$-point and at the edges of the Brillouin zone the doublets of levels are formed due to level repulsion. The eigenfunctions corresponding to the components of the doublet are either symmetric or antisymmetric functions of coordinates. Since at normal incidence only the symmetric mode can be excited, the antisymmetric mode turns out to be a deaf mode [20,21]. At normal incidence, a sharp minimum in the transmission spectrum appears when the frequency of the external wave coincides with the frequency of the symmetric component, which turns out to be the lower level of the doublet.

Excitation of the eigenmodes by an external plane wave is possible since the spectrum of eigenfrequencies is complex, i.e. all the eigenmodes of a linear chain are leaky modes radiating acoustic energy into the background fluid. An excited eigenmode transmits energy along the chain, i.e. the initial flux of energy is partially redirected by $90^{\circ}$. The effect of redirection of acoustic energy was recently predicted not only for periodic systems of scatterers $[16,19,22]$ but also for a narrow fluid channel in a solid elastic plate [23, 24].

Resonant interaction of the external wave with both eigenmodes - symmetric and antisymmetric - can be realized at oblique incidence. Even at small angles of incidence, the symmetry of the incoming front is broken by a nonzero component of the wave vector $\mathbf{k}_{||}$along the chain which allows excitation of the antisymmetric eigenmode. Excitation of any eigenmode in the chain requires matching of the Bloch vector of this eigenmode $\mathbf{q}$ and the parallel component $\mathbf{k}_{||}$of the wave vector in the incoming wave. The direction of propagation of acoustic energy is given by the vector of group velocity. For the symmetric mode possessing anomalous dispersion the redirected energy propagates against $\mathbf{k}_{\|}$, and for the antisymmetric mode with normal dispersion the energy runs in the direction of $\mathbf{k}_{\|}$. Since at small angles of incidence the matching condition $\mathbf{k}_{\|}=\mathbf{q}$ is satisfied for close frequencies, a chain of perforated shells may serve as a splitting antenna that redirects the higher-frequency harmonic of incoming signals along the direction of $\mathbf{k}_{\|}$and the lowerfrequency harmonic in the opposite direction. Our calculations show that anomalous scattering, redirection, and splitting of sound are robust with respect to viscosity. In particular, the viscosity of air does not undermine the pattern of anomalous scattering.

\section{SCATTERING PROBLEM FOR A FINITE-LENGTH LINEAR CHAIN OF PERFORATED CYLINDRICAL SHELLS}

Let a plane wave be scattered by a line of $2 N+1$ evenly spaced cylindrical shells. The shells' axes are centered along the $y$-axis at the points $y_{n}=n d, n=$ $0, \pm 1, \pm 2, \ldots, \pm N$. The wave vector $\mathbf{k}=\left(k_{x}, k_{y}, 0\right)$ of the pressure plane wave $p(\mathbf{r}, t)=p_{0} \exp (i \mathbf{k} \cdot \mathbf{r}-i \omega t)$ makes an angle $\theta$ with axis $x$, see Fig. 1 . The cylindrical shells are produced by rolling up perforated plates. Within the interval of parameters which we will use, the impedance of a flat plate perforated by circular holes can be approximated by [25]

$$
\begin{aligned}
Z_{p}=- & \frac{i \omega \rho_{0}}{\sigma}\left[h\left(1-\frac{2}{s \sqrt{i}} \frac{J_{1}(s \sqrt{i})}{J_{0}(s \sqrt{i})}\right)^{-1}\right. \\
& \left.+4 i \sqrt{2} \delta+\frac{16 r}{3 \pi}\left(1-2.5 \sqrt{\frac{\sigma}{\pi}}\right)\right] .
\end{aligned}
$$

Here $h=a-b$ is the thickness of the shell with $a$ and $b$ being the outer and inner radii respectively, $\sigma$ is the perforation filling fraction, and $s=r / \delta=r \sqrt{\rho_{0} \omega / \eta_{0}}$ is the perforate constant, which takes large values, $s \gg$ 1 , due to low viscosity $\eta_{0}$ and relatively large radius of perforations $r$. In the limit of an ideal fluid (inviscid, $s=\infty$ ) the impedance (1) becomes pure imaginary

$$
Z_{p}=-\frac{i \omega \rho_{0}}{\sigma}\left[h+\frac{16 r}{3 \pi}\left(1-2.5 \sqrt{\frac{\sigma}{\pi}}\right)\right] .
$$

While Eq. (2) was originally derived for a flat perforated plate, it turns out to be valid for cylindrical shells as well provided the wavelength exceeds the diameter of the perforations. Scattering by a perforated shell and by a shell with effective impedance (2) was studied numerically and experimentally in Refs. [26-28]. In each of these publications a good agreement was reported for a wide range of frequencies.

In numerical calculations, we use the same material parameters that were proposed in experimental and numerical study in Refs. $[19,27]$, namely: $a=4.00 \mathrm{~cm}$, 

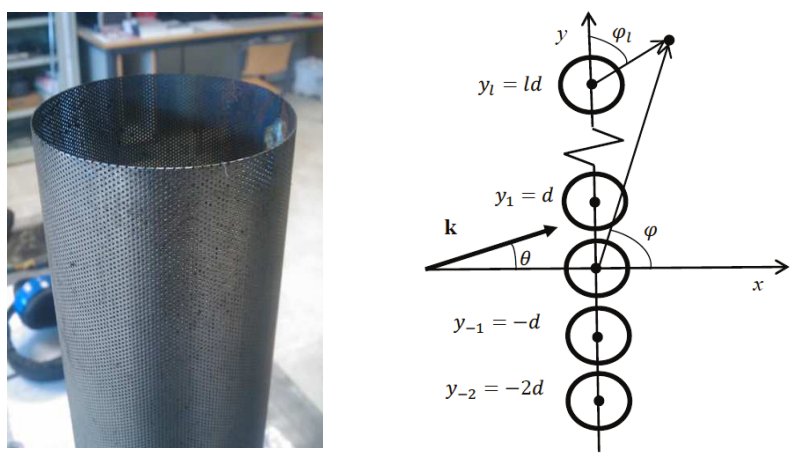

FIG. 1: Left panel: A perforated cylindrical shell fabricated and studied in Refs. [19, 26, 27]. Right panel: Perforated cylinders aligned along the $y$-axis with period $d$. Polar angles are shown for the central cylinder $(l=0)$ and for the $l$ th cylinder. The Cartesian coordinates $\mathbf{r}=(x, y)$ are related to the polar coordinates $\left(r_{l}, \varphi_{l}\right)$ associated with the center of the lth cylinder: $x=r_{l} \cos \varphi_{l}, y=y_{l}+r_{l} \sin \varphi_{l}$.

$h=0.05 \mathrm{~cm}, d=11.0 \mathrm{~cm}, r=0.25 \mathrm{~mm}, \sigma=14.5 \%$, $\rho_{0}=1.25 \mathrm{~kg} / \mathrm{m}^{3}$ (air), $c_{0}=343 \mathrm{~m} / \mathrm{s}$ (speed of sound in air), and $\eta_{0}=17.8 \mu \mathrm{Pa} \cdot s$ (air).

Scattering of waves by an infinite periodic chain of cylinders is one of the classical problems of theory of diffraction. For conducting cylinders illuminated by an electromagnetic wave the expansion of the scattered field was proposed in Ref. [8]. For sound waves, the field distribution resulting from diffraction in a periodic array of solid spheres in fluid was calculated in Ref. [29]. A similar problem for elastic waves scattered by a monolayer of elastic spheres was solved in Ref. [30]. In these studies, the chain of scatterers was considered to be infinite, i.e. the Bloch theorem was applicable. Here, we first calculate the acoustic field scattered by a finite-length chain. A periodic infinite chain is analyzed in the next section.

The incoming pressure plane wave $p(\mathbf{r}, t)$ can be expanded over cylindrical waves. Using the relation between the Cartesian and polar set of coordinates centered at $y=y_{n}$ shown in Fig. 1 we obtain

$$
\begin{aligned}
& p(\mathbf{r}, t)=p_{0} \exp (i \mathbf{k} \cdot \mathbf{r}-i \omega t)=p\left(r_{l}, \varphi_{l}, t\right) \\
& =p_{0} e^{i k r_{l} \cos \left(\varphi_{l}-\theta\right)-i \omega t}=p_{0} \sum_{n=-\infty}^{\infty} i^{n} J_{n}\left(k r_{l}\right) e^{i n\left(\varphi_{l}-\theta\right)-i \omega t} .
\end{aligned}
$$

Here $k=\omega / c_{0}$. The factor $e^{-i \omega t}$ is omitted in further calculations. The field scattered by a system of shells is written as a superposition of outgoing cylindrical waves radiated by each scatterer. We write this field in polar coordinates centered at $x=y=0$

$$
p_{s c}(r, \varphi)=\sum_{l^{\prime}} \sum_{n=-\infty}^{+\infty} B_{l^{\prime} n} H_{n}\left(k r_{l^{\prime}}\right) e^{i n \varphi_{l^{\prime}}}, \quad r_{l}^{\prime} \geq a .
$$

Here index $l^{\prime}$ numerates the shells in the chain and $H_{n}$ denotes the Hankel function of the first kind. If the chain is infinite, summation over $l^{\prime}$ runs from $-\infty$ to $\infty$. Otherwise, the sum includes only a finite number of terms. The relation between the polar coordinates $r=r_{0}, \varphi=\varphi_{0}$ and $r_{l}, \varphi_{l}$ is obtained from Fig. 1,

$$
r \cos \varphi=r_{l} \cos \varphi_{l}, r \sin \varphi=l d+r_{l} \sin \varphi_{l} .
$$

To express the scattered field in terms of polar coordinates of the system centered at the $l$ th cylinder $\left(r_{l}, \varphi_{l}\right)$ we apply Graf's addition theorem

$$
\begin{aligned}
& H_{n}\left(k r_{l^{\prime}}\right) e^{i n \varphi_{l^{\prime}}}=\sum_{n^{\prime}=-\infty}^{\infty} i^{\left(n+n^{\prime}\right) \operatorname{sign}\left(l-l^{\prime}\right)} H_{n+n^{\prime}}\left(k\left|l-l^{\prime}\right| d\right) \\
& J_{n^{\prime}}\left(k r_{l}\right) e^{i n^{\prime}\left(\pi-\varphi_{l}\right)} .
\end{aligned}
$$

It is valid for $l^{\prime} \neq l$ and $r_{l}<\left|l-l^{\prime}\right| d$. Now the scattered field $p_{s c}$ in Eq. (4) can be rewritten in terms of only one pair of coordinates $r_{l}, \varphi_{l}\left(r_{l}<d\right)$, linked to the position of the $l$ th cylinder

$$
\begin{aligned}
& p_{s c}\left(r_{l}, \varphi_{l}\right)=\sum_{n=-\infty}^{+\infty}\left[B_{l n} H_{n}\left(k r_{l}\right) e^{i n \varphi_{l}}+\sum_{l^{\prime} \neq l} B_{l^{\prime} n}\right. \\
& \left.\sum_{n^{\prime}=-\infty}^{+\infty} i^{\left(n+n^{\prime}\right) \operatorname{sign}\left(l-l^{\prime}\right)} H_{n+n^{\prime}}\left(k\left|l-l^{\prime}\right| d\right) J_{n^{\prime}}\left(k r_{l}\right) e^{i n^{\prime}\left(\pi-\varphi_{l}\right)}\right] .
\end{aligned}
$$

Pressure inside the $l$ th cylinder is expanded over the Bessel functions

$$
p_{i n}\left(r_{l}, \varphi_{l}\right)=\sum_{n=-\infty}^{\infty} C_{l n} J_{n}\left(k r_{l}\right) e^{i n \varphi_{l}} .
$$

The values of pressure in the incident wave (3), scattered wave (7), and the wave which penetrates inside the shells (8) are connected through the boundary conditions. For a thin perforated shell characterized by acoustic impedance $Z_{p}$ the normal component of velocity remains continuous at the fluid-solid boundaries. The value of the velocity itself is proportional to the jump discontinuity of the pressure,

$$
\left.v_{r}\right|_{r=a}=\left.v_{r}\right|_{r=b}=\frac{\left.p\right|_{r=b}-\left.p\right|_{r=a}}{Z_{p}}
$$

Here the radial velocity in fluid is

$$
v_{r}=-\frac{i}{\omega \rho_{0}} \frac{\partial p}{\partial r} .
$$

The velocity at the outer, $r_{l}=a$, and inner, $r_{l}=b$, shell's surfaces is given by pressure $p+p_{s c}$ and $p_{\text {in }}$, respectively.

Since the pressure in Eqs. (3), (7), and (8) is presented in the form of Fourier expansions over $e^{i n \varphi_{l}}$, the boundary conditions (9) can be written for the Fourier coefficients. This leads to the following set of linear equations which now does not contain angle $\varphi_{l}$ : 


$$
\left\{\begin{array}{l}
\left.p_{0} i^{n} e^{-i n \theta}+B_{l n} \frac{H_{n}^{\prime}(k a)}{J_{n}^{\prime}(k a)}+\sum_{l^{\prime} \neq l} \sum_{n^{\prime}=-\infty}^{\infty} i^{\left(n-n^{\prime}\right) \operatorname{sign}\left(l-l^{\prime}\right)} H_{n-n^{\prime}}\left(k\left|l-l^{\prime}\right|\right) d\right) B_{l^{\prime} n^{\prime}}=C_{l n} \frac{J_{n}^{\prime}(k b)}{J_{n}^{\prime}(k a)} \\
\left.p_{0} i^{n} e^{-i n \theta}+B_{l n} \frac{H_{n}(k a)}{J_{n}(k a)}+\sum_{l^{\prime} \neq l} \sum_{n^{\prime}=-\infty}^{\infty} i^{\left(n-n^{\prime}\right) \operatorname{sign}\left(l-l^{\prime}\right)} H_{n-n^{\prime}}\left(k\left|l-l^{\prime}\right|\right) d\right) B_{l^{\prime} n^{\prime}}=C_{l n} \frac{J_{n}(k b)}{J_{n}(k a)}+\frac{i k Z_{p}}{\omega \rho_{0}} C_{l n} \frac{J_{n}^{\prime}(k b)}{J_{n}(k a)}
\end{array}\right.
$$

For calculation of the scattered field only the coefficients $B_{l n}$ are necessary. Eliminating the unknown $C_{n l}$ from equations (11) we get the set of linear inhomogeneous equations for $B_{l n}$

$$
\begin{aligned}
& \mathcal{S}_{n} B_{l n}+\sum_{l^{\prime} \neq l} \sum_{n^{\prime}=-\infty}^{\infty} i^{\left(n-n^{\prime}\right) \operatorname{sign}\left(l-l^{\prime}\right)} H_{n-n^{\prime}}\left(k\left|l-l^{\prime}\right| d\right) B_{l^{\prime} n^{\prime}} \\
& =-p_{0} i^{n} e^{-i n \theta}
\end{aligned}
$$

where

$$
\mathcal{S}_{n}=\frac{H_{n}(k a)-H_{n}^{\prime}(k a)\left(\frac{i Z_{p}}{\rho_{0} c_{0}}+\frac{J_{n}(k b)}{J_{n}^{\prime}(k b)}\right)}{J_{n}(k a)-J_{n}^{\prime}(k a)\left(\frac{i Z_{p}}{\rho_{0} c_{0}}+\frac{J_{n}(k b)}{J_{n}^{\prime}(k b)}\right)} .
$$

Solution of the linear set (12) gives the distribution of the scattered acoustic field Eq. (6) for any finite number of shells.

This set of equations was solved numerically for different number of cylinders $2 N+1$ in the chain. Then the transmission coefficient

$$
T(f)=\frac{1}{2 N d p_{0} v_{0}} \int_{-N d}^{N d} p_{t o t}(d, y) v_{t o t, x}^{*}(d, y) d y
$$

was calculated and plot in Fig. 2. Here $p_{t o t}(x, y)=p+p_{s c}$ and $v_{t o t, x}(x, y)=-\left(i / \omega \rho_{0}\right) \partial p_{t o t} / \partial x$ are the the total pressure and the $x$-component of the total velocity, respectively. The transmission spectrum was calculated for inviscid air [see Eq. (2)] and for air with its real viscosity. In accordance with the numerical results obtained in Ref. [19] the transmission coefficient is very close to one within a wide range of frequencies. The only minimum is observed around $3 \mathrm{kHz}$. It is demonstrated in the next section that this minimum is due to resonant coupling of the incident wave to one of the eigenmodes of the chain. Viscosity reduces the deepness of the minimum and also leads to visible red shift of the resonance.

Since the scattering at perforated shells is quite weak, the spectrum of eigenmodes is established for a sufficiently long chain. Otherwise, the collective behavior of the eigenmodes is weakly manifested which leads to a very shallow minimum in the transmission. The spectra for relatively small number of shells are shown in Fig. 3. For a chain of 15 cylinders the minimum is hardly seen, while here the viscosity is neglected. In short chains, adding the viscosity will completely undermine

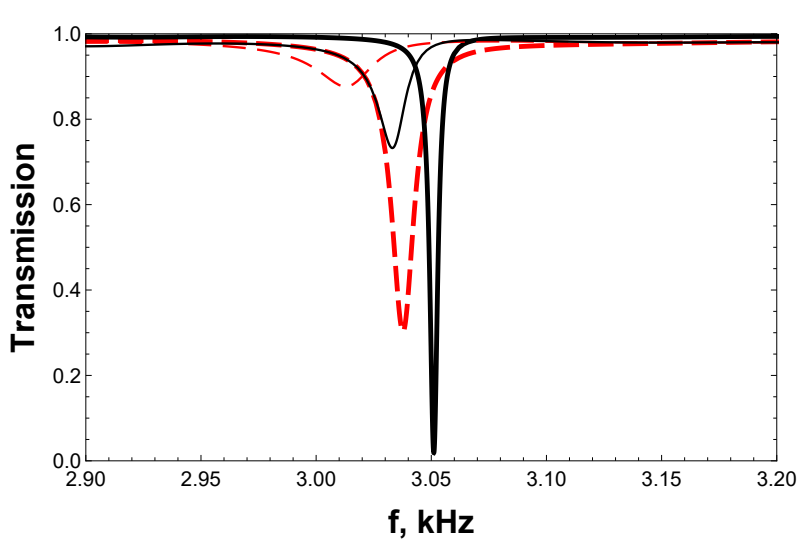

FIG. 2: Transmission coefficient for the chain of 31 (dashedred lines) and 41 (solid-black lines) perforated shells. Results for inviscid and viscous air are shown by thick and thin lines respectively.

the resonance. The resonant minimum becomes deeper and sharper with the number of scatterers. The position of the minimum is blue-shifted approaching $3078 \mathrm{~Hz}$ which is the first non-zero frequency at the $\Gamma$-point in the $1 \mathrm{D}$ band structure of the infinite periodic chain of shells imbedded in inviscid air. It is interesting to note that the deepness of the minimum is less sensitive to the number of scatterers than the position of the resonance. This can be seen from the inserts to Fig. 3 where the transmission at minimum approaches zero faster than the resonant frequency approaches its limiting value of $3078 \mathrm{~Hz}$.

\section{SCATTERING BY AN INFINITE PERIODIC CHAIN}

For infinite periodic chain of scatterers the Bloch's theorem is applicable. Then, the unknowns $B_{l n}$ are related to the values $B_{0 n}$ associated with the central shell at $y=0$

$$
B_{l n}=e^{i k_{y} l d} B_{0 n}, \quad k_{y}=k \sin \theta
$$

Substituting this relation to Eq. (12) and renormalizing the unknowns leads to the following set of linear equa- 


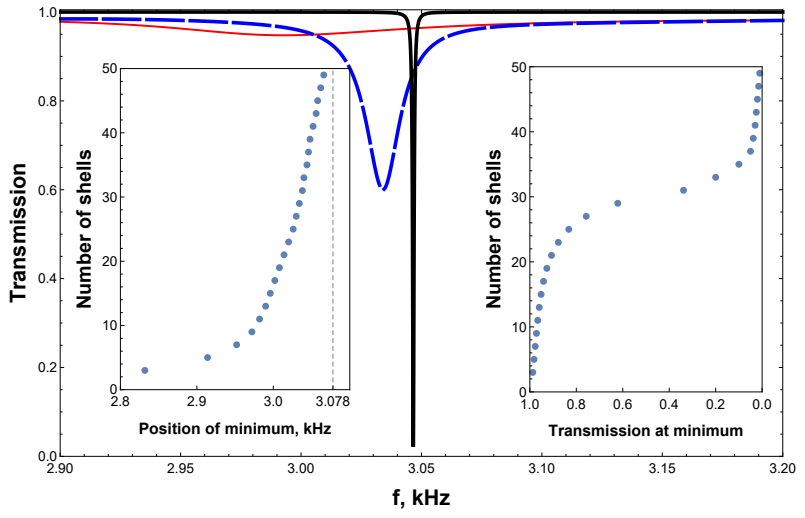

FIG. 3: Transmission coefficient for the chain of 15 (solid-red line), 29 (dashed-blue line), and 37 (solid-black line) perforated shells. Results are obtained for inviscid air. Inserts: position (left panel) of the transmission minimum and its deepness (right panel) vs number of shells in the chain.

tions for the coefficients $b_{n}=i^{-n} B_{0 n}$ :

$\mathcal{S}_{n} b_{n}+\sum_{n^{\prime}=-\infty}^{\infty} F\left(n^{\prime}-n\right) b_{n}^{\prime}=-p_{0} e^{-i n \theta}, n=0, \pm 1, \pm 2, \ldots$

Here

$$
F(n)=\sum_{l^{\prime}=1}^{+\infty} H_{n}\left(k l^{\prime} d\right)\left[e^{i k_{y} l^{\prime} d}+(-1)^{n} e^{-i k_{y} l^{\prime} d}\right] .
$$

A similar set of equations was obtained in Ref. [8] for scattering of electromagnetic wave at an infinite periodic chain of metallic cylinders.

The eigenmodes of an infinite chain of shells are the nontrivial solutions of Eq. (16) in absence of incident wave $\left(p_{0}=0\right)$. Thus, the dispersion relation $\omega=\omega(q)$, where $q=k_{y}$, is obtained by equating to zero the determinant of the corresponding homogeneous linear set

$$
\operatorname{det}\left|\mathcal{S}_{n} \delta_{n n^{\prime}}+F\left(n^{\prime}-n\right)\right|=0
$$

Direct calculation of the matrix elements $F\left(n^{\prime}-n\right)$ requires summation of slowly converging series of cylindrical functions in Eq. (17). It was shown in Ref. [31] that these series can be identically replaced by the series with fast convergence. The necessary formulas, which also were used in Ref. [8], are given in Appendix A.

The numerical solution of this dispersion equation for the few lowest bands is presented in Fig. 4. Since perforated shells are weak scatterers, the dispersion curve (blue dots) is very close to the band structure obtained in the empty-lattice model, shown by the straight broken line (red). Only at the $\Gamma$-point $q=0$ (and at the edge $q=\pi / d$ ) the effect of level repulsion leads to gap opening of $35 \mathrm{~Hz}$ and essentially nonlinear dispersion, see the left insert to Fig. 4. It is shown in Appendix B that near the point of degeneracy, $f=3078 \mathrm{~Hz}$, the upper and lower

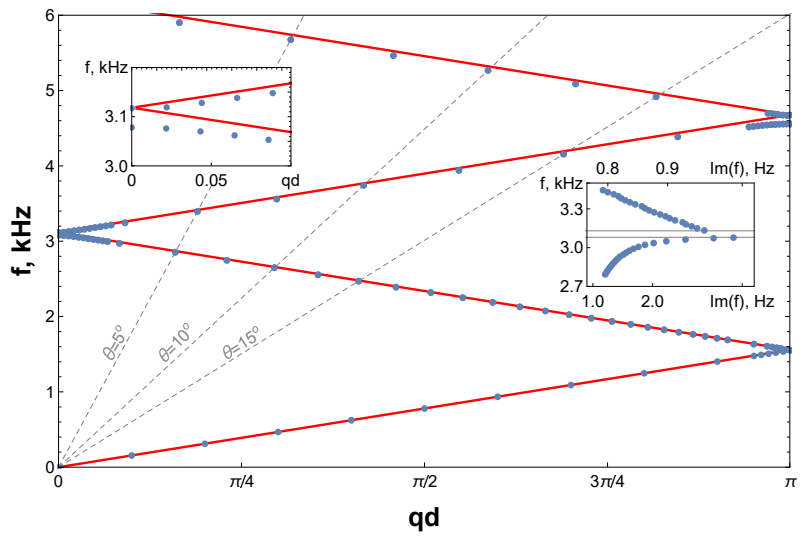

FIG. 4: Band structure of an infinite periodic chain of shells imbedded in inviscid air. Solid straight (red) lines show linear dispersion in air. Dots (blue) are the real parts of eigenfrequencies calculated from Eq. (18). Crossings of the dispersion curves with three dashed straight lines gives the frequencies of resonant coupling of the incident plane wave to the eigenmodes of the chain for three angles of incidence, $\theta=5^{\circ}$, $\theta=10^{\circ}$, and $\theta=15^{\circ}$. Inserts: (left) band splitting at the $\Gamma$-point near the frequency $f=3 \mathrm{kHz}$. The Wood's anomalies are observed at these resonant frequencies. The distance between the levels in the doublet is $35 \mathrm{~Hz}$; (right) Absolute value of the imaginary part of eigenfrequency vs its real part for the region near $3 \mathrm{kHz}$. The horizontal axes, where the imaginary part of the frequency is plot, are different for the upper and lower levels of the doublet.

bands exhibit different behavior. In particular, the gap opening is strongly asymmetric and the group velocity vanishes at $q=0$ only for the lower band. There are also some singularities in the dispersion of the upper band which are not resolved in Fig. 4. They are shown in the insert to Fig. 10. Away from the $\Gamma$-point the dispersion of the both bands is practically linear but it is normal for the upper band and anomalous for the lower one.

For real values of the wavevector $q$ all the solutions $\omega(q)=2 \pi f(q)$ of the transcendental equation (16) are complex. This means that the acoustic eigenmodes of an infinite chain of shells are leaky modes, even if the viscosity of the fluid is neglected. The imaginary part of frequency describes weak radiative decay, i.e. the acoustic eigenmodes are not strongly localized near the chain. However, since the decay is very slow the leaky eigenmodes are long-living excitations. The imaginary part is negative, $\operatorname{Im} f<0$, in order for the time-dependent factor $e^{-i \omega t}$ to decay with time. The rate of radiative decay $|\operatorname{Im} f|$ is at least two orders of magnitude smaller than the frequency of sound $f \approx \operatorname{Re} f$ as it can be seen from the right insert to Fig. 4. For the frequencies near the $\Gamma$-point the rate of radiative decay is about $1-3 \mathrm{~Hz}$, i.e. it practically does not contribute to the width of the resonance minima observed in Figs. 2 and 3. The plots in Figs. 2 and 3 show that the width of the resonance is defined mainly by viscosity and by the length of the 


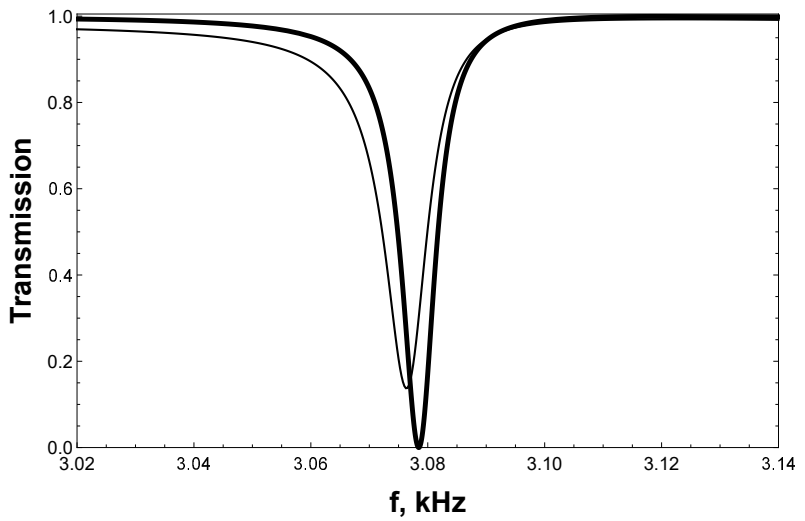

FIG. 5: Transmission spectrum of an infinite chain of perforated cylindrical shells at normal incidence. Thick and thin lines are for inviscid and viscous air respectively.

chain. The minimum of the width of resonance, $\Delta f=30$ $\mathrm{Hz}$, is observed for the ideal situation of infinite chain and inviscid air, see the thick line in Fig. 5.

The transmission vanishes exactly at $f=3078 \mathrm{~Hz}$, which is the frequency of the lower level in the doublet. Numerical results show that the pressure is distributed symmetrically (antisymmetrically) over the $y$-axis for the eigenstates belonging to the lower (higher) level of the doublet. Only symmetric modes can be excited by external plane wave at normal incidence. The upper level, being an antisymmetric mode, cannot be excited and for this geometry it is referred to as deaf mode [20, 21]. Direct excitation of the symmetric mode in one-dimensional chain of scatterers leads to practically $100 \%$ suppression of transmission, with about $85 \%$ of energy being reflected back and $15 \%$ of energy being evenly redirected by $\pm 90^{\circ}$ into two opposite directions along the chain.

In a sonic crystal of elastic rods proposed in Ref. [16] the redirection of sound occurs due to the Poisson-like effect. The effect deals with excitation of one of the antisymmetric deaf modes, which becomes accessible due to its coupling to flexural resonances of a rod through an evanescent mode. Since in this case the excitation is indirect, maximum $46 \%$ of acoustic energy is reflected or redirected at the frequency of the mid-gap.

It is important to note that the resonance in a chain embedded in a viscous air is only weakly broadened and slightly red shifted by dissipation, see the thin line in Fig. 5. Because of viscosity the transmission at the resonance becomes finite while still about $80 \%$ of acoustic energy is either reflected or redirected due to excitation of the symmetric leaky modes of the chain. Since the leaky modes are not proper spectral modes, they interact with the environment and can be excited by external sound. Also, since $\operatorname{Im} f \ll \Delta f \ll \operatorname{Re} f$ the resonant excitation is well established.

\section{ANOMALOUS SCATTERING AT OBLIQUE INCIDENCE}

At oblique incidence the symmetry of the incoming wave is broken and both eigenmodes (symmetric and antisymmetric) can be excited. Therefore, the transmission spectra in Fig. 6 exhibit two minima. Each minimum is observed at the frequency where the matching condition for the wave vectors

$$
k_{y}=\left(2 \pi f / c_{0}\right) \sin \theta=q(f)
$$

is satisfied. Matching of the tangential components of the wave vectors is known as the manifestation of Wood's anomaly, see, e.g., Ref. [34]. In the reduced zone scheme this condition is satisfied for several frequencies. For example, in Fig. 4 this condition is satisfied at the points of crossing of two straight dashed lines, corresponding to the angles of incidence $\theta=5^{\circ}$ and $\theta=15^{\circ}$, with the dispersion curves. For $\theta=5^{\circ}$ two crossings are shown at $f \approx 2.85 \mathrm{kHz}$ and $f \approx 3.40 \mathrm{kHz}$. The transmission minima in Fig. 6 occur near these frequencies. The frequency interval between these minima is about $550 \mathrm{~Hz}$ that significantly exceeds the width of each minimum as well as the width of the doublet at the $\Gamma$-point. That is why each minimum is well-resolved. Strong separation of the minima is due to different dispersion of the upper and lower bands, which are the continuations of the levels of the doublet at the $\Gamma$-point. For the upper (antisymmetric) band the dispersion is normal, whereas it is anomalous for the lower (symmetric) band, i.e. the phase and group velocities of this eigenmode have opposite directions.

At normal incidence, $\theta=0^{\circ}$, Eq. (19) is formally satisfied at any non-zero frequency where the dispersion curve in Fig. 4 crosses the vertical line $q=0$. However, the minimum in the transmission spectrum (Wood's anomaly) appears only at the frequencies corresponding to the symmetric modes. Since the dispersion curve in Fig. 4 is very close to the linear dispersion in water, the Wood's anomaly in Fig. 5 is observed at the wavelength of $11.1 \mathrm{~cm}$ that practically coincides with the period of the chain, $d=11 \mathrm{~cm}$.

Due to different dispersion, the scattering of incident waves by a chain of perforated cylinders exhibits an interesting anomaly. For both eigenmodes the direction of their phase velocities coincides with the direction of the wave vector $\mathbf{q}=q \hat{y}$, which is obtained from Eq. (19). However, the group velocity for the lower band is opposite to $\mathbf{q}$ that leads to scattering of the incoming wave in the "wrong" direction.

The angular distribution of intensity of scattered sound for $\theta=5^{\circ}$ is plot in Figs. 7 and 8. In Fig. 7, the frequency of the incoming wave is $3.40 \mathrm{kHz}$ that allows coupling with the upper band shown in Fig. 4. Since this band has normal dispersion, the maximum of the scattered field is pointed towards the direction of the parallel component $k_{y} \hat{y}$ of the wave vector in the incoming wave. For the frequency $f=2.85 \mathrm{kHz}$, the matching condition 


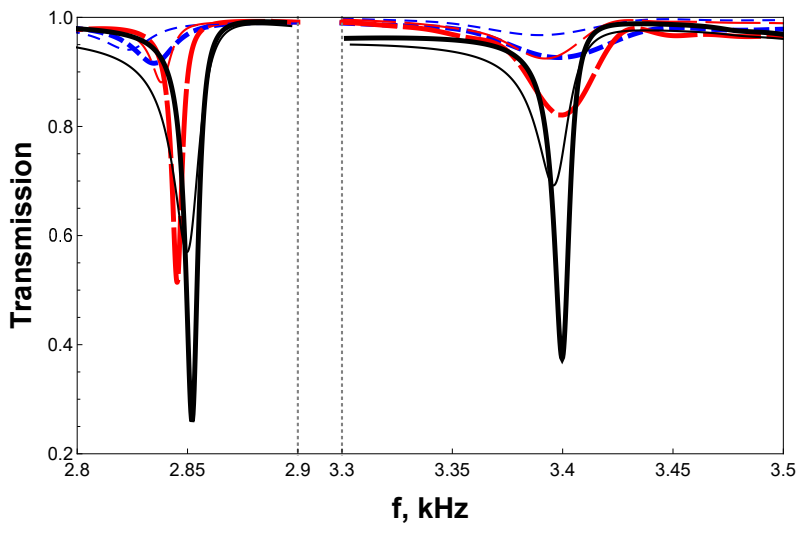

FIG. 6: Transmission spectrum at oblique incidence, $\theta=5^{\circ}$. Blue-short-dashed, red-long-dashed, and black solid lines are for the chains containing 61, 121, and infinite number of cylindrical shells, respectively. Thick and thin lines show the results for inviscid and viscous air. Note that two resonances are separated by $\approx 550 \mathrm{~Hz}$ in frequency, that exceeds much the width of the doublet $(\approx 35 \mathrm{~Hz})$.

(19) is satisfied for the lower band with anomalous dispersion. As a result, the maximum of intensity points against $k_{y} \hat{y}$, thus producing anomalous pattern for the scattered field in Fig. 8. Viscosity of air reduces the intensity of the scattered field in all the directions. Polar diagrams for viscous air are shown by green-dashed lines in Figs. 7 and 8. It is clear that the coupling to the eigenmodes remains effective, leading to the well-pronounced peaks in the normal and "wrong" directions.

For the angles of incidence $\theta \geq 5^{\circ}$ the intensities of the normal and anomalous scattering do not differ much. This can be seen also from the values of the transmission coefficients in the minima in Fig. 5 where the lower-frequency dip is only $10 \%$ stronger than the higherfrequency one. For angles smaller than $5^{\circ}$ the anomalous scattering dominates since the eigenmode with normal dispersion cannot be excited at $\theta=0^{\circ}$ because of its antisymmetric nature. When $\theta \rightarrow 0^{\circ}$ the frequency interval between two resonant minima in the transmission spectra approaches the width of the doublet, while at the same time the minimum corresponding to normal scattering gradually vanishes.

The plots in Figs. 5-8 clearly demonstrate that a periodic chain of weak scatterers may redirect the incoming sound beam by an angle of about $90^{\circ}$ if the frequency and the angle of incidence satisfy the matching condition (19). Moreover, a chain may serve as a splitter of bifrequency acoustic signal. Let an acoustic beam formed by mixing of two monochromatic components hit a chain at a certain angle $\theta$. If the frequencies match the condition (19), the beam will be split into two monochromatic signals propagating along the chain in the opposite directions. If the detuning between the components is quite small (beats) the crossing of the straight line with the dispersion curves in Fig. 4 occurs very close to the $\Gamma$ -

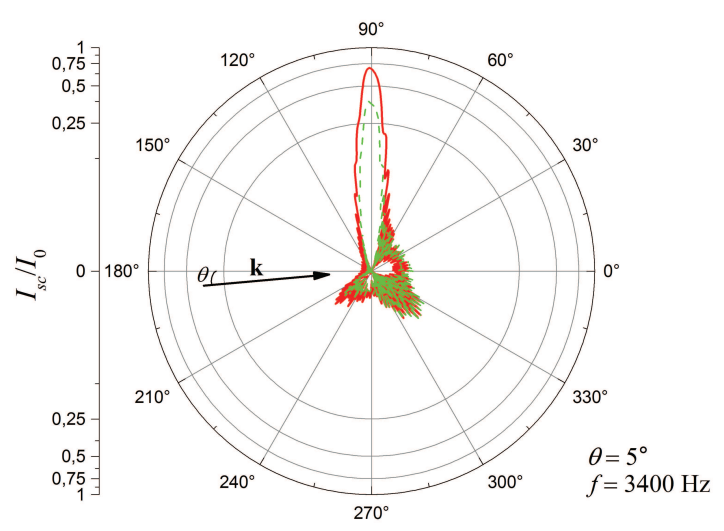

FIG. 7: Polar diagram showing distribution of intensity of scattered sound of frequency $f=3400 \mathrm{~Hz}$ (upper band). Solid-red and green-dashed lines are for inviscid and viscous air, respectively. Black arrow shows the direction of the incident wave. An essential part of scattered sound wave propagates along the chain in the direction of the wave vector $\mathbf{k}_{\|}$. Note logarithmic scale along radius.

point, that corresponds to almost normal incidence. In this case, the efficiency of splitting is reduced since the intensity of the redirected high-frequency component is suppressed.

In Fig. 9 we show the splitting of a bi-frequency signal when it hits the chain at the angle of $10^{\circ}$ in ideal (panel (a)) and viscous (panel (b)) air. The frequencies of the monochromatic components, $2625 \mathrm{~Hz}$ and $3715 \mathrm{~Hz}$, are obtained from the band diagram in Fig. 4. Since the chain contains only 25 shells and the angle of incidence is not very small, the essential part of acoustic energy propagates directly through the chain. Nevertheless, a clear pattern of split fringes is observed even for viscous air. The efficiency of splitting is quite good for a pure mechanical splitter, for ideal (viscous) air 8\% (5\%) of energy is converted into the low-frequency component, propagating down (anomalous scattering) and 10\% (7\%) of energy is converted into the higher-frequency component, propagating up (normal scattering).

\section{CONCLUSIONS}

In summary, we have demonstrated redirection and splitting of sound waves impinging a periodic chain of thin perforated cylindrical shells. These conclusions have been obtained using an analytical approach to the problem of diffraction of sound by a periodic chain of perforated cylindrical shells, which has been developed here. Scattering at the shells is described in impedance approximation, which is applicable for both lossless and viscous background fluid. The dispersion equation for the eigenmodes of an infinite periodic chain is derived and its complex solutions are obtained numerically for 


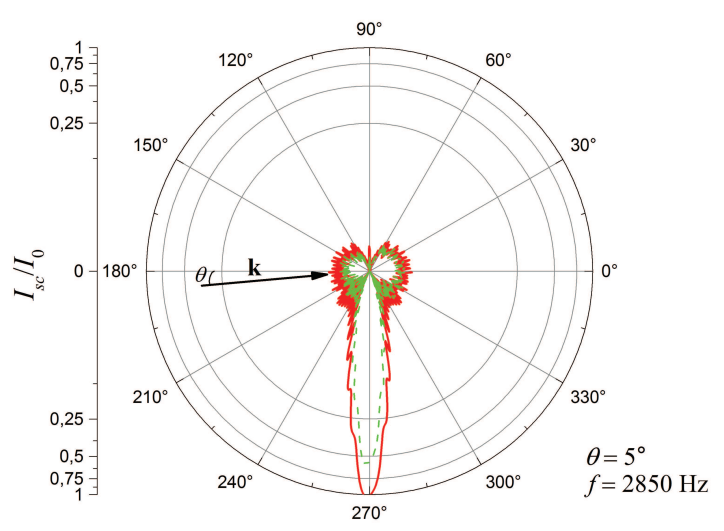

FIG. 8: The same as in Fig. 7 but for the frequency $f=$ $2850 \mathrm{~Hz}$ that corresponds to the lower band with anomalous dispersion. In this case the scattering occurs in the "wrong" direction, i.e. opposite to $\mathbf{k}_{\|}$.

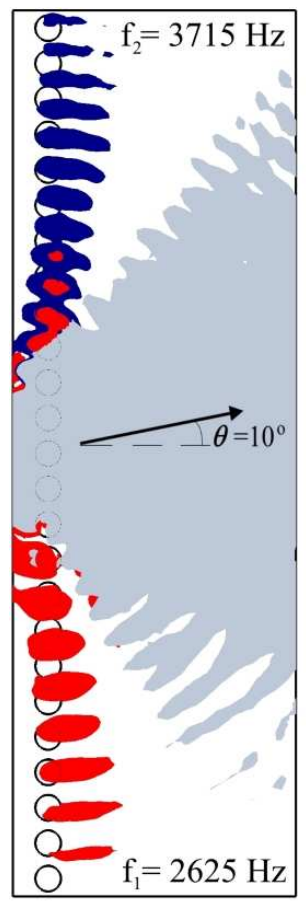

a)

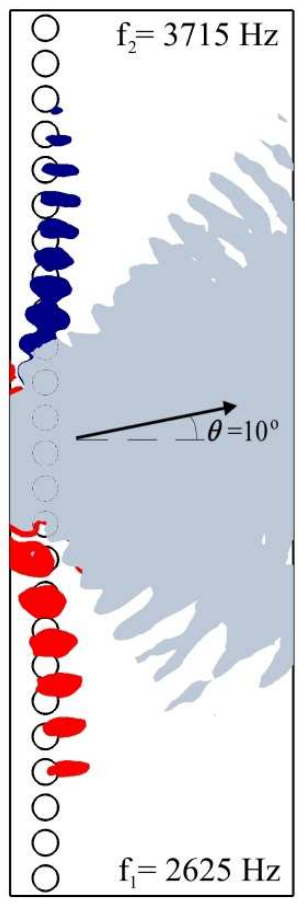

b)
FIG. 9: Distribution of intensity of bi-frequency signal (with $f_{1}=2625 \mathrm{~Hz}$ and $f_{2}=3715 \mathrm{~Hz}$ ) transmitted through a chain of 25 perforated shells. The angle of incidence is $10^{\circ}$ and the background air is inviscid (a) and viscous (b). The central part of the diffraction pattern (shown in grey) is a mixture of two sound waves with frequencies $f_{1}$ and $f_{2}$. The red and blue fringes are the split monochromatic components. The low-frequency component (red) exhibits anomalous scattering, propagating against the "natural" direction. aluminum perforated shells in air. Since these shells are only weak scatterers of sound, the eigenmodes are leaky waves propagating with phase velocity close to the speed of sound in air, and they slowly decay due to dissipation and radiation. The transmission coefficient is calculated for infinite and finite-length chains within a wide range of frequencies. At normal incidence, the coupling to the lowest symmetric eigenmode gives rise to a deep minimum in the transmission. In this geometry, the antisymmetric mode, which is close in frequency, is not excited and the incoming wave is equally redirected along the chain in both directions. At oblique incidence, the external wave can be coupled to both symmetric and antisymmetric modes that leads to two deep minima in the transmission spectrum which are well-resolved for viscous air. Here anomalous scattering is strongly manifested because the symmetric mode exhibits anomalous dispersion while for the antisymmetric one the dispersion is normal. We find that resonant coupling of external sound to the symmetric mode results in strong scattering of acoustic energy along the chain in the "wrong" direction with respect to the direction of incidence. Finally, we show that due to different dispersion of symmetric and antisymmetric modes a sound beam consisting of two (or more) monochromatic components can be effectively split into two parts propagating in opposite directions along the chain. Each part is a monochromatic wave with the frequency resonating with either symmetric or antisymmetric eigenmode. Further experimental work should be performed in order to support our theoretical predictions, which foresee useful devices for the filtering and splitting of sound waves at selected frequencies.

\section{ACKNOWLEDGEMENT}

A.K. acknowledges support from Programa de Apoyo a la Investigacion y Desarrollo (PAID-02-15) de la Universitat Politècnica de València. A. B., F. C. and J. S.-D. acknowledge the support by the Ministerio de Economía y Competitividad of the Spanish government, and the European Union FEDER through project TEC2014-53088C3-1-R. 
[1] M. Quinten, A. Leitner, J.R. Krenn, and F.R. Aussenegg, Electromagnetic energy transport via linear chains of silver nanoparticles, Opt. Lett. 23, 1331 (1998).

[2] M.L. Brongersma, J.W. Hartman, and H.A. Atwater, Electromagnetic energy transfer and switching in nanoparticle chain arrays below the diffraction limit, Phys. Rev. B 62, R16356 (2000).

[3] S.A. Maier, P.G. Kik, H.A. Atwater, S. Meltzer, E. Harel, B.E. Koel, A.A.G. Requicha, Local detection of electromagnetic energy transport below the diffraction limit in metal nanoparticle plasmon waveguides, Nat. Mater. 2, 229 (2003).

[4] W.H. Weber and G.W. Ford, Propagation of optical excitations by dipolar interactions in metal nanoparticle chains, Phys. Rev. B 70, 125429 (2004).

[5] V.A. Markel and A.K. Sarychev, Propagation of surface plasmons in ordered and disordered chains of metal nanospheres, Phys. Rev. B 75, 085426 (2007).

[6] C. Tserkezis, N. Papanikolaou, E. Almpanis, and N. Stefanou, Tailoring plasmons with metallic nanorod arrays, Phys. Rev. B 80, 125124 (2009).

[7] P. Jasper Compaijen, Victor A. Malyshev, and Jasper Knoester, Surface-mediated light transmission in metal nanoparticle chains, Phys. Rev. B 87205437 (2013).

[8] Sergey Belan and Sergey Vergeles, Plasmon mode propagation in array of closely spaced metallic cylinders, Optical Materials Express, 5, 130 (2015).

[9] Adam Brandstetter-Kunc, Guillaume Weick, Charles A. Downing, Dietmar Weinmann, and Rodolfo A. Jalabert, Nonradiative limitations to plasmon propagation in chains of metallic nanoparticles, Phys. Rev. B 94, 205432 (2016).

[10] H.H. Huang and C.T. Sun, Wave attenuation in an acoustic metamaterial with negative effective mass density, New J. Phys. 11, 013003 (2009).

[11] H.H. Huang and C.T. Sun, Anomalous wave propagation in one-dimensional acoustic metamaterial having simaltaneously negative mass density and Youg's modulus, J. Acoust. Soc. Am. 132, 2887 (2012).

[12] N. Kaina, F. Lemoult, M. Fink and G. Lerosey, Negative refractive index and acoustic superlens from multiple scattering in single negative metamaterials, Nature 525, 77 (2015).

[13] Michael R. Haberman and Matthew D. Guild, Acoustic metamaterials, Phys. Today 69(6), 42 (2016).

[14] Steven A. Cummer, Johan Christensen, and Andrea Alú, Controlling sound with acoustic metamaterials, Nat. Rev. Mater. 1, 16001 (2016).

[15] T. P. Martin, C.N. Layman, K. M. Moore and G. J. Orris, Elastic shells with high-contrast material properties as acoustic metamaterial components, Phys. Rev. B 85, 161103(R) (2012).

[16] A. S. Titovich and A. N. Norris, Acoustic Poisson-like effect in periodic structures, J. Acoust. Soc. Am. 139, 3353 (2016).

[17] A. S. Titovich and A. N. Norris, Tunable cylindrical shell as an element in acoustic metamaterial, J. Acoust. Soc. Am. 136, 1601 (2014).

[18] A. S. Titovich and A. N. Norris, Acoustic scattering from an infinitely long cylindrical shell with an internal mass attached by multiple axisymmetrically distributed stiffeners, J. Sound. Vib. 338, 134 (2015).

[19] V. M. García-Chocano and J. Sánchez-Dehesa, Anomalous sound absorption in lattices of cylindrical perforated shells, Appl. Phys. Lett. 106, 124104 (2015); J. SánchezDehesa, V. M. García-Chocano, D. Torrent, F. Cervera, S. Cabrera and F. Simon, Noise control by sonic crystal barriers made of recycled materials, J. Acoust. Soc. Am., 129, 1173 (2011).

[20] J. V. Sánchez-Pérez, D. Caballero, R. Mártinez-Sala, C. Rubio, J. Sánchez-Dehesa, F. Meseguer, J. Llinares, and F. Gálvez, Sound attenuation by a two-dimensional array of rigid cylinders, Phys. Rev. Lett. 80, 5325 (1998).

[21] G. P. Ward, A. P. Hibbins, J. R. Sambles and J.D. Smith, Acoustic transmission through compound subwavelength slit arrays, Phys. Rev. B 94, 024304 (2016).

[22] Bart van der Aa and Jens Forssén, Upward refraction of sound propagating outdoors by a graded index sonic crystal noise barrier, Applied Acoustics 74, 89 (2013).

[23] V. M. García-Chocano, Nagaraj, T. López-Rios, L. Gumen, J. Sánchez-Dehesa, and A. Krokhin, Resonant coupling of Rayleigh waves through a narrow fluid channel causing extraordinary low acoustic transmission, J. Acoust. Soc. Am. 132, 2807 (2012).

[24] A. Bozhko, V. M. García-Chocano, J. Sánchez-Dehesa, and A. Krokhin, Redirection of sound in straight fluid channel with elastic boundaries, Phys. Rev. B 91, 094303 (2015).

[25] Dah-You Maa, Potential of microperforated panel absorber, J. Acoust. Soc. Am. 104, 2861 (1998).

[26] V. M. García-Chocano, S. Cabrera, and J. SánchezDehesa, Broadband sound absorption by lattices of microperforated cylindrical shells, Appl. Phys. Lett. 101, 184101 (2012).

[27] V. M. García-Chocano, Ph.D. thesis, New devices for noise control and acoustic cloaking, Universitat Politècnica de València, 2015.

[28] Bart van der Aa and Jens Forssén, Scattering by an array of perforated cylinders with a porous core, J. Acoust. Soc. Am. 136, 2370 (2014).

[29] Y. Zhang and P. Wei, The scattering of acoustic wave by a chain of elastic spheres in liquid, Journal of Vibration and Acoustics 136, 021023 (2014).

[30] R. Sainidou, N. Stefanou, I. E. Psarobas, and A. Modinos, Scattering of elastic waves by a periodic monolayer of spheres, Phys. Rev. B 66, 024303 (2002).

[31] V. Twersky, Elementary function representation of the Schlömilch series, Arch. Rational Mech. Anal. 8, 323 (1961).

[32] T. C. Lim and G. W. Farnell, Character of pseudo surface waves on anisotropic crystals, J. Acoust. Soc. Am. 45, 845 (1969).

[33] K. A. Ingebrigtsen and A. Tonning, Elastic surface waves in crystals Phys. Rev. 184, 942 (1969).

[34] Daniel Maystre, Theory of Wood's Anomalies, in Plasmonics, Springer Series in Optical Sciences 167, edited by S. Enoch and N. Bonod, (Springer-Verlag Berlin Hei- 
delberg, 2012) p. 39.

[35] N.E. Glass and A.A. Maradudin, Leaky surface-elastic waves on both flat and strongly corrugated surfaces for isotropic, non dissipative media, J. Appl. Phys. 54, 796 (1983).

[36] A.A. Maradudin and I. Simonsen, Rayleigh and Wood anomalies in the diffraction of acoustic waves from the periodically corrugated surface of an elastic medium, Low Temperature Physics, 42, 354 (2016).

[37] A.A. Maradudin, I. Simonsen, and W. Zierau, Rayleigh and Wood anomalies in the diffraction of light from a perfectly conducting reflection grating, Optics Lett. 41, 2229 (2016).

\section{Appendix A: Lattice sum}

The lattice sum (17) converges slowly since $\left|H_{n}(k l d)\right| \propto 1 / \sqrt{k l d}$ for $l \gg 1$. It was shown in Ref. [31] that this lattice sum can be rewritten in the following equivalent form with much faster convergence:

$$
\begin{gathered}
F(0)=-1-\frac{2 i}{\pi}\left[\gamma+\ln \frac{k}{2 p}\right]-\frac{2 i\left(k^{2}+2 q^{2}\right)}{p^{3} d} \zeta(3)-\frac{2 i}{\gamma_{0} d}-\frac{2 i}{d} \sum_{m=1}^{+\infty}\left(\frac{1}{\gamma_{m}}+\frac{1}{\gamma_{-m}}-\frac{2}{m p}-\frac{k^{2}+2 q^{2}}{m^{3} p^{3}}\right), \\
F(2 n)=-\frac{2 i e^{-2 i n \alpha_{0}}}{\gamma_{0} d}-2 i \sum_{m=1}^{+\infty}\left(\frac{e^{-2 i n \alpha_{m}}}{\gamma_{m} d}+\frac{e^{2 i n \alpha_{-m}}}{\gamma_{-m} d}-\frac{(-1)^{n}}{m \pi}\left(\frac{k}{2 m p}\right)^{2 n}\right)- \\
-\frac{2 i(-1)^{n}}{\pi}\left(\frac{k}{2 p}\right)^{2 n} \zeta(2 n+1)+\frac{i}{n \pi}+\frac{i}{\pi} \sum_{m=1}^{n} \frac{(-1)^{m} 2^{2 m}(n+m-1) !}{(2 m) !(n-m) !}\left(\frac{p}{k}\right)^{2 m} \mathcal{B}_{2 m}\left(\frac{q}{p}\right), \\
F(2 n-1)=\frac{2 i e^{-i(2 n-1) \alpha_{0}}}{\gamma_{0} d}+2 i \sum_{m=1}^{+\infty}\left(\frac{e^{-i(2 n-1) \alpha_{m}}}{\gamma_{m} d}-\frac{e^{i(2 n-1) \alpha_{-m}}}{\gamma_{-m} d}+\frac{i(-1)^{n} q d n}{m^{2} \pi^{2}}\left(\frac{k}{2 m p}\right)^{2 n-1}\right)+ \\
+\frac{2 i(-1)^{n} q d n}{\pi^{2}}\left(\frac{k}{2 p}\right)^{2 n-1} \zeta(2 n+1)-\frac{2}{\pi} \sum_{m=0}^{n-1} \frac{(-1)^{m} 2^{2 m}(n+m-1) !}{(2 m+1) !(n-m-1) !}\left(\frac{p}{k}\right)^{2 m+1} \mathcal{B}_{2 m+1}\left(\frac{q}{p}\right) .
\end{gathered}
$$

Here $\mathcal{B}_{m}$ is the Bernoulli polynomial, $\gamma=0.577$ is Euler's constant and

$$
p=\frac{2 \pi}{d}, \quad q_{m}=q+m p, \quad \gamma_{m}=-i \sqrt{k^{2}-q_{m}^{2}}, \quad \alpha_{m}=\arcsin \frac{q_{m}}{k} .
$$

These equations are true for $n \geq 0$ and for any complex $k$ with non-negative imaginary part. For $n<0$, obviously, $f_{-n}=(-1)^{n} f_{n}$. When $\operatorname{Im} k<0$, the original series (17) exponentially diverges. Eqs. (A1)-(A3) can be considered as analytic continuation of the original function $F(n)$ to the lower semispace in the complex $k$ plane. Also, in the case of complex values of $k$, the branch cut defining the square root in $\gamma_{m}$ must be properly chosen. The particular choice of the branch cut will be discussed in Appendix B below.

\section{Appendix B: Perturbation theory}

Assuming that the perforated shells are weak scatterers, the solution of the dispersion equation (18) can be found using a perturbation theory. In zero approximation, the dispersion that corresponds to the empty-lattice model is linear. Corrections to this linear dispersion are of interest only near the points of degeneracy, i.e. in the vicinity of the $\Gamma$-point and the edges of the Brillouin zone. In the theory of band structures, these corrections are usually obtained using perturbation theory for degenerate states. Due to weak scattering, the degeneracy is lifted, giving rise to narrow band gaps. In solids, this kind of perturbation theory is known as the nearly free electron model and it uses the basis of plane waves as unperturbed eigenfunctions. Here, we look for the solution of the wave equation in the basis of cylindrical functions that leads to strong modification of the perturbation theory as compared to the well-known nearly free electron model. While the final result is the same - opening of narrow band gaps, it is worthwhile to get the explicit formulas for the gap width and for the level repulsion near the points of degeneracy and to compare these formulas with the results obtained nonperturbatively from the exact dispersion equation (18). It will be shown that because of the specific form of the boundary condition (9), which assumes a discontinuity of pressure even for very thin shell, the strength of the level repulsion is quite different from that observed in the nearly free electron model. 
The scattering of sound by perforated cylinders vanishes when the acoustic impedance $Z_{p} \rightarrow 0$. This situation is different from the case of solid cylinders when absence of scattering requires matching of the impedance of the material of the cylinders and the impedance $c_{0} \rho_{0}$ of the background fluid. Thus, the small parameter of the perturbation theory is impedance $Z_{p}$ that requires that the thickness of the shell $h=a-b \rightarrow 0$. The latter condition is necessary but not sufficient, as it is seen from Eqs. (1) and (2).

The coefficient $\mathcal{S}_{n}$ in Eq. (13) approaches infinity when $a \rightarrow b$ and $Z_{p} \rightarrow 0$, so we rewrite the dispersion relation (18) in the form:

$$
\operatorname{det}\left|\delta_{n n^{\prime}}+\frac{F\left(n^{\prime}-n\right)}{\mathcal{S}_{n}}\right|=0
$$

Since $F\left(n^{\prime}-n\right) / \mathcal{S}_{n} \ll 1$, the determinant of almost diagonal matrix in Eq. (B1) can be approximated by

$$
\operatorname{det}\left|\delta_{n n^{\prime}}+\frac{F\left(n^{\prime}-n\right)}{\mathcal{S}_{n}}\right| \approx \operatorname{det}\left|\delta_{n n^{\prime}}\right|+\operatorname{Tr}\left(\frac{F\left(n^{\prime}-n\right)}{\mathcal{S}_{n}}\right)=1+F(0) \sum_{n=-\infty}^{+\infty} \mathcal{S}_{n}^{-1}
$$

The terms $\mathcal{S}_{n}^{-1}$ in the series (B2) can be expanded over $h=a-b$ and $Z_{p}$. In the linear approximation, we obtain

$$
\begin{aligned}
\mathcal{S}_{n}^{-1}= & \frac{J_{n}(k a)-J_{n}^{\prime}(k a)\left(\frac{i Z_{p}}{\rho_{0} c_{0}}+\frac{J_{n}(k b)}{J_{n}^{\prime}(k b)}\right)}{H_{n}(k a)-H_{n}^{\prime}(k a)\left(\frac{i Z_{p}}{\rho_{0} c_{0}}+\frac{J_{n}(k b)}{J_{n}^{\prime}(k b)}\right)} \\
\approx & \frac{\pi k a}{2 i} \frac{i Z_{p}}{\rho_{0} c_{0}} J_{n}^{\prime 2}(k a)-\frac{1}{2 i} \pi k^{2} a(a-b)\left(J_{n}^{\prime 2}(k a)-J_{n}(k a) J_{n}^{\prime \prime}(k a)\right),
\end{aligned}
$$

It follows from Eq. (B3) that the expansion goes over two real (for inviscid fluid) dimensionless parameters $z=\frac{\pi k a}{2} \frac{i Z_{p}}{\rho_{0} c_{0}}$ and $\epsilon=\pi k^{2} a h$. Here $k=\omega / c_{0}$. Since $Z_{p} \propto \omega$ (see Eq. (1)) the parameters $z, \epsilon \propto \omega^{2}$. Because of this quadratic growth the approximation (B3) becomes invalid at high frequencies.

Substitution of the expansion (B3) into the sum over $n$ in Eq. (B2) gives the following result:

$$
\begin{aligned}
\sum_{n=-\infty}^{+\infty} \mathcal{S}_{n}^{-1} & =\frac{\pi k a}{2 i} \frac{i Z_{p}}{\rho_{0} c_{0}} \sum_{n=-\infty}^{+\infty} J_{n}^{\prime 2}(k a)-\frac{1}{2 i} \pi k^{2} a h \sum_{n=-\infty}^{+\infty}\left(J_{n}^{\prime 2}(k a)-J_{n}(k a) J_{n}^{\prime \prime}(k a)\right)= \\
& =\frac{\pi k a}{4 i} \frac{i Z_{p}}{\rho_{0} c_{0}}-\frac{1}{2 i} \pi k^{2} a h=\frac{z-\epsilon}{2 i}
\end{aligned}
$$

where the identities $\sum_{n=-\infty}^{+\infty} J_{n}^{\prime 2}(x)=-\sum_{n=-\infty}^{+\infty} J_{n}(x) J_{n}^{\prime \prime}(x)=\frac{1}{2}$ were used. Now Eqs. (B1) and (B2) can be simplified to

$$
\frac{1}{F(0)}=-\sum_{n=-\infty}^{+\infty} \mathcal{S}_{n}^{-1}=-\frac{z-\epsilon}{2 i}
$$

In zero approximation, the right-hand side of Eq. (B5) vanishes, therefore the dispersion is obtained from the condition $F(0)=\infty$. It can be satisfied due to the resonant terms with $\gamma_{m}=-i \sqrt{k^{2}-q_{m}^{2}}$ in the denominator in Eq. (A1). One of the terms diverges if for any integer $m$

$$
\omega=c_{0}|q \pm 2 \pi m / d|, \quad m=0,1,2, \ldots
$$

This recovers the linear dispersion for sound obtained in the empty lattice approximation.

In the next approximation over $z$ and $\epsilon$ the same resonant terms are left in the lattice sum

$$
F(0) \approx-\sum_{m^{\prime}=-\infty}^{\infty} \frac{2 i}{\gamma_{m} d} \approx \frac{2}{d} \sum_{m^{\prime}=-\infty}^{\infty} \frac{1}{\sqrt{k^{2}-\left(q+2 \pi m^{\prime} / d\right)^{2}}}
$$

Looking for the level splitting near the $\Gamma$-point, the parameter $k=\omega / c_{0}$ is represented as $k=|2 \pi m / d|+\Delta k_{m}$, where $\Delta k_{m}$ is a small correction. 
The eigenmodes of a chain of perforated cylinders are leaky waves since the dispersion relation $f(q)$ shown in Fig. 4 contains small negative imaginary part leading to exponential decay of the field amplitude at $t \rightarrow \infty$. At the same time, the function $F(n)$ defined by Eq. (17) becomes singular in the lower complex $k$ plane since the sum over $l^{\prime}$ diverges when the complex argument of the Hankel function infinitely grows. This follows from the asymptotical behavior $H_{n}\left(k l^{\prime} d\right) \approx \sqrt{2 /\left(\pi k l^{\prime} d\right)} \exp \left(i k l^{\prime} d-i \pi n / 2-i \pi / 4\right)$. The function $F(n)$ can be analytically continued to the region $\operatorname{Im} k<0$ by fast convergent series (A1)-(A3), provided that a single-valued branch of $\sqrt{k^{2}-q_{m}^{2}}$ is defined.

While the expansion of the scattered field (7) runs over cylindrical waves, the parameter $\gamma_{m}=\sqrt{k^{2}-q_{m}^{2}}$ serves (far away from the chain) as a perpendicular to the chain component of the wave vector. A leaky mode with $\operatorname{Re}\left(k^{2}-q_{m}^{2}\right)>0$ and $\operatorname{Im}\left(k^{2}-q_{m}^{2}\right)<0$ (fourth quadrant) radiates towards $x>0$. The corresponding exponent $e^{i \gamma_{m} x}$ oscillates with exponentially growing amplitude, i.e. $\operatorname{Re} \gamma_{m}>0$ and $\operatorname{Im} \gamma_{m}<0$ (fourth quadrant). Increase of the amplitude "compensates" exponential decay of the energy of leaky mode along the direction of propagation [32, 33, 35]. For the square root $\sqrt{k^{2}-q_{m}^{2}}$ with its argument $k^{2}-q_{m}^{2}$ lying in the forth quadrant, the branch of the square root lying also in the same quadrant is defined if the cut is made, e.g., along the negative imaginary axis. This choice is made, among other options, because it also provides the correct behavior for the non-radiative (true) eigenmodes [36, 37]. Indeed, for the non-radiative wave $\operatorname{Im} k<0$ and $\operatorname{Re}\left(k^{2}-q_{m}^{2}\right)<0$, so $\left(k^{2}-q_{m}^{2}\right)$ lies in the third quadrant. Then its square root lies either in the second or fourth quadrant. The non-radiative condition requires that $\operatorname{Im} \sqrt{k^{2}-q_{m}^{2}}>0$. This condition uniquely defines the branch of the square root in the second quadrant and the branch cut along the negative imaginary axis.

Once the unique branch for $\gamma_{m}$ is defined, the dispersion equation (B5) with $F(0)$ in the form (B7) can be solved. For a given allowed band with band index $m$ only two terms with $m^{\prime}=m$ and $m^{\prime}=-m$ are left in Eq. (B7) in order to calculate the repulsion of levels near the $\Gamma$-point (near the edge of the Brillouine zone the terms with $m^{\prime}=m$ and $m^{\prime}=-m-1$ give the principal contribution). In this region where $k=\omega / c_{0} \approx 2 \pi m / d$ and $q \ll \pi / d$ the denominators in Eq. (B7) can be simplified to $\sqrt{k^{2}-q_{ \pm m}^{2}} \approx \sqrt{(4 \pi m / d)\left(\Delta k_{m} \mp q\right)}$. Thus, in the linear approximation the dispersion equation takes the following form:

$$
\frac{1}{\sqrt{\Delta k_{m}-q}}+\frac{1}{\sqrt{\Delta k_{m}+q}}=-i \sqrt{4 \pi m d}(z-\epsilon)^{-1}
$$

This equation has a unique solution provided that the both terms in the left-hand side are pure imaginary. Since $q>0$ the correction $\Delta k_{m}$ must be negative and also $\left|\Delta k_{m}\right|>q$. These conditions define the lower branch of the dispersion curve near $\omega=2 \pi m c_{0} / d$. It is therefore easy to write down the equation for $\left|\Delta k_{m}\right|$ as

$$
\sqrt{\left|\Delta k_{m}\right|+q}+\sqrt{\left|\Delta k_{m}\right|-q}=2 \sqrt{\frac{\Delta k_{m}^{2}-q^{2}}{\kappa_{m}}},
$$

where $\kappa_{m}=(z-\epsilon)^{2} / \pi m d$. This equation is valid for any value of the Bloch vector $q<\left|\Delta k_{m}\right|$. To calculate the level repulsion it is sufficient to solve this equation in the close vicinity of the $\Gamma$-point, where $q \ll\left|\Delta k_{m}\right|$. Exactly at the $\Gamma$-point $\Delta k_{m}(q=0)=\kappa_{m}$. Expansion of the both parts of Eq. (B9) up to the terms $\propto q^{2}$ reduces it to a quadratic equation over $\left|\Delta k_{m}\right|$. Its physically meaningful solution is

$$
\Delta k_{m}=-\kappa_{m}-\frac{3 q^{2}}{4 \kappa_{m}} .
$$

Using this result, the dispersion relation for the lower branch is written as follows:

$$
\omega(q)=\frac{2 \pi m c_{0}}{d}+\Delta k_{m} c_{0} \approx \frac{2 \pi m c_{0}}{d}-\kappa_{m} c_{0}-\frac{3 q^{2}}{4 \kappa_{m}} c_{0} .
$$

This dispersion relation looks similar to what is known from the nearly free electron approximation: the group velocity vanishes at the $\Gamma$-point, the eigenfrequency is red-shifted with respect to that in the empty lattice model, and the width of the band gap $\kappa_{m} c_{0}$ scales as a square of the perturbation parameter. Note that the obtained dispersion relation is real since the non-radiative condition $k^{2}-q_{ \pm m}^{2}<0$ is valid. The imaginary correction to the eigenfrequency appears when the terms with $\left|m^{\prime}\right| \neq m$ are kept in the sum (B7).

Unlike this, the upper branch of the dispersion curve is obtained as a complex solution of Eq. (B8). According to the requirement of exponential decay with time, this solution lies in the lower part of the complex plane, $\operatorname{Im} \Delta k_{m}=$ $\operatorname{Im} \omega / c_{0}<0$. Since the right-hand-side of Eq. (B8) is pure imaginary, the real parts of $\left(\Delta k_{m}-q\right)$ and $\left(\Delta k_{m}+q\right)$ have opposite signs, i.e. the former lies in the third and the latter in the fourth quadrant. Then, the two inequalities $\operatorname{Re}\left(\Delta k_{m}+q\right)>0$ and $\operatorname{Re}\left(\Delta k_{m}-q\right)>0$ yield $\left|\operatorname{Re} \Delta k_{m}\right|<q$, i.e. the complex solution of Eq. (B8) does not reach 
the $\Gamma$-point $q=0$. Taking into account the branch cut along the negative imaginary axis, the first and the second term in Eq. (B8) lie in the third and first quadrants, respectively. Introducing dimensionless variables $x=q / \kappa_{m}$ and $y=\Delta k_{m} / \kappa_{m}$ Eq. (B8) can be rewritten as follows:

$$
\frac{1}{\sqrt{y-x}}=-2 i-\frac{1}{\sqrt{y+x}}
$$

Both sides of this radical equation lie in the same (third) quadrant, therefore it can be safely squared. Repeating this procedure two times, we come to a biquadratic equation over $x$

$$
4 x^{4}+x^{2}\left(1-8 y^{2}-4 y\right)+4 y^{4}+4 y^{3}=0 .
$$

While it can be solved exactly, we are interested in the dependence $y(x)$ in the region of small $x$ and $y$. It is easy to get that $y(x)$ can be written as a power series over $(x / 2)^{2 / 3}$

$$
y(x) \approx e^{-i \pi / 3}\left(\frac{x}{2}\right)^{2 / 3}+\frac{5}{3} e^{i \pi / 3}\left(\frac{x}{2}\right)^{4 / 3}+\left(\frac{x}{2}\right)^{2}, x<<1 .
$$

Now the dispersion relation for the upper branch is written as follows:

$$
\omega(q)=\frac{2 \pi m c_{0}}{d}+\Delta k_{m} c_{0} \approx \frac{2 \pi m c_{0}}{d}+\kappa_{m} c_{0}\left[e^{-i \pi / 3}\left(\frac{q}{2 \kappa_{m}}\right)^{2 / 3}+\frac{5}{3} e^{i \pi / 3}\left(\frac{q}{2 \kappa_{m}}\right)^{4 / 3}+\left(\frac{q}{2 \kappa_{m}}\right)^{2}\right]
$$

This relation is valid for small wavevectors, $q<<\kappa_{m}$. It, however, is not valid very close to the $\Gamma$-point where the group velocity $\partial \omega / \partial q \propto q^{-1 / 3}$ exhibits infinite growth. The region of validity is obtained from the condition Re $\Delta k_{m}<q$, which numerically gives $q>q_{c} \approx \kappa_{m} / 20$. Within the interval $0<q<q_{c}$, shown by dashed line in the insert to Fig. 10, the mode does not propagate. This region is so narrow that it is not resolved in the dispersion curves for the upper branch shown in Figs. 4 and 10. Unlike the lower band, which exhibits the red-shift, the upper branch is not blue-shifted, and the gap opening

$$
\Delta f=\frac{c_{0} \kappa_{1}}{2 \pi}=\frac{c_{0}(z-\epsilon)^{2}}{2 \pi^{2} d},
$$

is asymmetric. Numerically the gap width $\Delta f \approx 100 \mathrm{~Hz}$ given by this perturbation theory formula exceeds much the band gap of only $35 \mathrm{~Hz}$ shown in Fig. 4. The reason is that the expansion in Eq. (B3) runs not over the ratio of impedances $i Z_{p} / \rho_{0} c_{0}$ but over the parameter $z=\frac{\pi k a}{2} \frac{i Z_{p}}{\rho_{0} c_{0}}$ which is not small at all. Near $\omega=2 \pi c_{0} / d$ its numerical value is as big as 0.95, that is why the perturbation theory gives a poor approximation. Nevertheless, this approximation is of principal importance since it explains the asymmetry between the lower and the upper band near the $\Gamma$-point.

The asymmetric band opening and non-vanishing group velocity of the upper band have been recently reported for leaky surface acoustic [36] and electromagnetic [37] modes propagating along periodically corrugated surfaces. The eigenvalue problem for surface modes at the surface corrugations, having cylindrical symmetry with axis along $z$, and the eigenvalue problem for the periodic set of cylinders in Fig. 1 require similar expansions over cylindrical functions. Due to this similarity the spectra of leaky eigenmodes for these two geometries exhibit the same anomalies. 


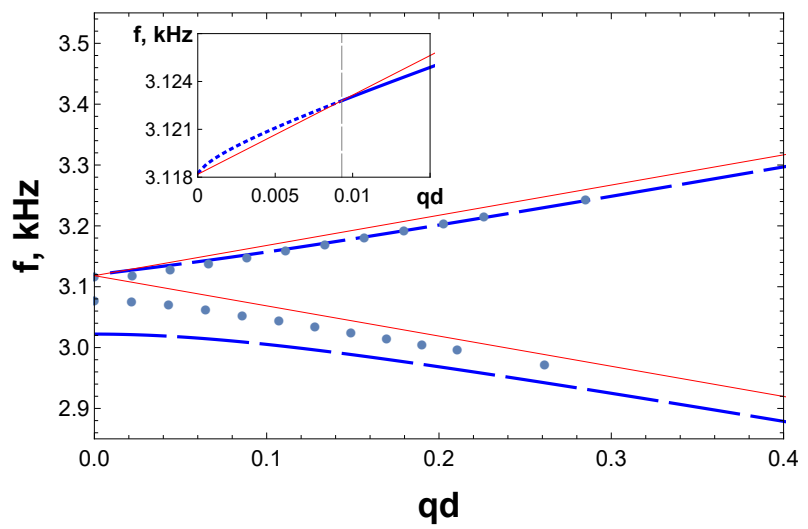

FIG. 10: Band structure of infinite periodic chain of shells imbedded in inviscid air. Solid straight (red) lines show linear dispersion in air. Dots (blue) are the real parts of the eigenfrequencies calculated from Eq. (18). Dashed (blue) lines show the dispersion obtained from the perturbation theory equations (B11) and (B15). The inset shows the narrow region $q d<q_{c} d \approx 0.01$ near the $\Gamma$-point where the upper band does not exist. The leaky upper mode beyond $q=q_{c}$ is shown by solid blue line. 